\title{
Kayseri'nin merkez ilçelerinde süs bitkilerinde bulunan yaprakbiti (Hemiptera: Aphididae) türleri ${ }^{1}$
}

\author{
Aphid (Hemiptera: Aphididae) species on ornamental plants in central districts of \\ Kayseri
}

\section{Deniz Özkan ÖZTÜRK ${ }^{2}$}

\author{
Murat MUŞTU²
}

Abstract

This study was carried out in central districts (Hacılar, İncesu, Kocasinan, Melikgazi and Talas) of Kayseri, in 2014-2015. Aphid specimens were collected from trees and ornamental plants in bush form in parks, roadsides and landscape areas in the districts. At the end of study, thirty aphid species belonging to 23 genera from 7 subfamilies; Anoeciinae, Aphidinae, Callaphidinae, Eriosomatinae, Lachninae, Mindarinae and Pterocommatinae, were determined. Because of there is not any record related with 14 of these species from Kayseri province, these species were reported as new record for Kayseri aphid fauna. These species are Anoecia (Anoecia) corni (Fabricius, 1775), Rhopalosiphum nymphaeae (Linnaeus, 1761), Rhopalosiphum rufiabdominale (Sasaki, 1899), Amphorophora (Amphorophora) rubi (Kaltenbach, 1843), Cavariella (Cavariella) aegopodii (Scopoli, 1763), Dysaphis (Dysaphis) crataegi (Kaltenbach, 1843), Liosomaphis berberidis (Fitch, 1851), Metopolophium (Metopolophium) dirhodum (Walker, 1849), Eucallipterus tilia (Linnaeaus, 1758), Myzocallis (Myzocallis) coryli (Goetze,1778), Cinara (Cubressobium) cupressi (Buckton, 1881), Lachnus roboris (Linnaeus, 1758), Mindarus abietinus Koch, 1857, Pterocomma pilosum Buckton, 1879. In addition, Prunus mahaleb L. has been identified as a new host plant for Myzus cerasi (Fabricius, 1775) in Turkey.

Keywords: Fauna, survey, ornamental plants, aphid

\section{Öz}

Bu çalışma 2014-2015 yıllarında Kayseri ili merkez ilçeleri (Hacılar, İncesu, Kocasinan, Melikgazi ve Talas)'nde yürütülmüştür. Materyal yaprakbiti örnekleri ilçelerdeki parklar, yol kenarı ve peyzaj alanlarında bulunan ağaçlar ve çalı formundaki süs bitkileri üzerinden toplanmıştır. Çalışma sonunda, Aphididae familyasının Anoeciinae, Aphidinae, Callaphidinae, Eriosomatinae, Lachninae, Mindarinae ve Pterocommatinae olmak üzere 7 altfamilyasına dahil olan 23 cinse ait 30 yaprakbiti türü tespit edilmiştir. Bu türlerden 14 tanesinin Kayseri ilinde kaydına rastlanılmadığı için, bu türler Kayseri yaprakbiti faunası için yeni kayıt olarak bildirilmiştir. Bu türler Anoecia (Anoecia) corni (Fabricius, 1775), Rhopalosiphum nymphaeae (Linnaeus, 1761), Rhopalosiphum rufiabdominale (Sasaki, 1899), Amphorophora (Amphorophora) rubi (Kaltenbach, 1843), Cavariella (Cavariella) aegopodii (Scopoli, 1763), Dysaphis (Dysaphis) crataegi (Kaltenbach, 1843), Liosomaphis berberidis (Fitch, 1851), Metopolophium (Metopolophium) dirhodum (Walker, 1849), Eucallipterus tilia (Linnaeaus, 1758), Myzocallis (Myzocallis) coryli (Goetze,1778), Cinara (Cubressobium) cupressi (Buckton, 1881), Lachnus roboris (Linnaeus, 1758), Mindarus abietinus Koch, 1857, Pterocomma pilosum Buckton, 1879'dir. Ayrıca, Prunus mahaleb L. Türkiye'de Myzus cerasi (Fabricius, 1775) için yeni konukçu bitki olarak tespit edilmiştir.

Anahtar sözcükler: Fauna, sürvey, süs bitkileri, yaprakbiti

\footnotetext{
1 Bu çalışma, ilk yazarın yüksek lisans tezinin bir bölümü olup, FYL-2014-5341 numaralı proje kodu ile Erciyes Üniversitesi Bilimsel Araştırma Projeleri Birimi tarafından desteklenmiş ve Türkiye VI. Bitki Koruma Kongresi'nde poster olarak sunulmuştur ${ }^{2}$ Erciyes Üniversitesi, Seyrani Ziraat Fakültesi, Bitki Koruma Bölümü, Kayseri

*Sorumlu yazar (Corresponding author) e-mail: mmustu77@hotmail.com

Alınış (Received): 24.11.2017 Kabul Ediliş (Accepted): 21.06.2018

Çevrimiçi Yayın Tarihi (Published Online): 10.09.2018
} 


\section{Giriş}

Süs bitkileri, genelde yerel veya ithal edilen tropik ağaç veya çalı formundaki bitkilerden oluşurlar. Bu bitkiler genelde, şehir merkezlerinde park ve oyun alanlarını süsledikleri gibi, yol ortasında veya yol kenarlarında görsel güzelliği sağlamak ve doğal canlı popülasyonlarını koruma amacı ile de kullanılırlar. Bunun için genelde çalı formundaki bitkiler ile çok yıllık iğne yapraklı ağaçlar tercih edilir. Fakat şehir merkezlerindeki parkların ağaçlandırılması amacıyla kullanılan bitki formları çok çeşitlilik gösterebilmektedir. Çeşitli bitkiler görsel güzelliği sunmasının yanı sıra, doğal yaşamın aksamadan sürmesine de yardımcı olmaktadır. Ayrıca, bu alanlar sehir merkezinde yaşam imkanı bulan böceklerin de önemli sığınak yerlerinden biridir. Kolayca üreyebildikleri, popülasyon oluşturup, dağılabildikleri ve her ne kadar ilaçlamalar ile bazen bozulsa da, doğal ekosistemdeki av-avcı ve parazit/parazitoit-konukçu ilişkisinin yoğun bir şekilde devam ettiği yerlerdir.

Süs bitkilerinin en yaygın ve en önemli zararılıarından olan yaprakbitleri; doğrudan bitkilerle beslenmeleri, hastalık etkenlerini taşımaları ve fumajine neden olmalarından dolayı, ekonomik açıdan önemli bir böcek grubudur. Dünyada şu ana kadar yaklaşık olarak 510 cinse ait 5000 yaprakbiti türü tespit edilmiştir (Blackman \& Eastop, 2017). Türkiye'de ise yaprakbiti faunası ile ilgili ilk çalışmalar 1900'lü yılların başında başlamış olup, günümüze kadar tespit edilen tür sayısı 541 tür ve 13 alttüre ulaşmıştır (Görür et al., 2017)

Şu ana kadar Kayseri il merkezinde yaprakbiti faunasının belirlenmesine yönelik kapsamlı olarak sadece tek bir çalışma yapıımıştır. Söz konusu çalışmada, Kayseri merkezdeki kültür bitkileri, süs bitkileri ve doğal olarak bulunan bitkiler üzerinden 6 tribus içerisinden 23 cinse bağlı 36 türe ait yaprakbiti örnekleri toplanmıştır (Şahin, 2007). Bu çalışmada, Kayseri ili merkez ilçelerindeki parklar, yol kenarları ve peyzaj alanlarındaki süs bitkilerinde bulunan yaprakbiti türlerinin belirlenmesi amaçlanmıştır.

\section{Materyal ve Yöntem}

\section{Örneklerin Toplanması ve Kültüre Alınması}

Örneklemeler 2014-2015 yılları Nisan-Ekim ayları arasında, periyodik olmayan arazi çıkışları yapılarak bitkilerin yaprak ve gövdeleri üzerinden toplanarak gerçekleştirilmiştir. Tüm merkez ilçelere her ay en az bir kez gidilerek arazi çalışması yapılmasına özen gösterilmiştir. Kış aylarında örnekleme yapılmamıştır.

Yaprakbiti ile bulaşık bitki organları budama makası ile kesilerek önce kese kağıdı, sonra polietilen naylon torbalara konularak ve etiketlenerek laboratuvara getirilmiştir. Laboratuvara getirilen örnekler içerisinde nimf döneminde olanlar ergin döneme ulaşıncaya kadar, üzerinde bulundukları bitki organı ile birlikte kültüre alınmışlardır. Ergin dönemde olanlar ile laboratuvarda ergin döneme ulaşan bireyler daha sonra \%70'lik alkol içerisine alınarak preparatı yapılmak üzere etiketlenmiştir. Yaprakbitlerinin preparasyonu Hille Ris Lambers (1950) yöntemine göre yapılmış ve örnekler Dr. Işıl Özdemir (Ankara Zirai Mücadele Merkez Araştırma Enstitüsü) tarafından teşhis edilmiştir.

\section{Konukçu bitkilerinin örneklenmesi}

Yaprakbitlerinin konukçusu olan bitkilere ait örnekler kese kağıdı içerisinde alınarak teşhisi yapılmak üzere saklanmıştır. Konukçu bitki örneklerinin teşhisleri Prof. Dr. Cem VURAL (Erciyes Üniversitesi, Biyoloji Bölümü) tarafından yapılmıştır.

\section{Araştırma Sonuçları ve Tartışma}

2014-2015 yılları arasında Kayseri ili merkez ilçelerindeki parklar, yol kenarları ve peyzaj alanlarındaki süs bitkilerinde yapılan sörveylerde Aphididae familyasının Anoeciinae, Aphidinae, Callaphidinae, Eriosomatinae, Lachninae, Mindarinae ve Pterocommatinae olmak üzere 7 altfamilyasına dahil 23 cinse ait 30 yaprakbiti türü tespit edilmiştir. 


\section{Familya: Aphididae}

\section{Altfamilya: Anoeciinae}

\section{Anoecia (Anoecia) corni (Fabricius, 1775)}

İncelenen materyal: Cornus mas üzerinde, Fuar Parkı (Kocasinan), 17.V.2014.

Zoocoğrafi Dağılımı: Afro-Tropikal, Palaearktik, Nearktik, Neotropikal ve Oryantal Bölge (Nieto Nafria, 2017).

Türkiye'deki Dağılımı: Ankara, Bitlis, Diyarbakır, Isparta, İstanbul, Mersin, Rize, Tekirdağ, Trabzon, Samsun (Tuatay \& Remaudiere, 1964; Çanakçıŏlu, 1966; Çanakçığlu, 1967; Çanakçıoğlu, 1975; Düzgüneş et al., 1982; Tuatay, 1999; Toros et al., 2002; Ölmez Bayhan et al., 2003; Görür et al., 2009; Akyürek, 2013; Akyıldııı et al., 2014; Barjadze et al., 2014). Kayseri faunası için yeni kayıttır.

\section{Altfamilya: Aphidinae}

\section{Tribe: Aphidini}

\section{Aphis (Aphis) craccivora Koch, 1854}

İncelenen materyal: Cercis siliquastrum üzerinden, Gazi Yusuf Tulgay Parkı (Talas), 18.V.2014; Adliye Parkı (İncesu) 10.V.2014, 21.VIII.2014; Erciyes Üniversitesi kampüsü (Melikgazi) 30.V.2015; Muhsin Yazıcıoğlu Parkı (Talas), 27.V.2014, 22.V.2015; Fraxinus ornus üzerinden, Hisarcık Meydan (Melikgazi), 18.V.2015; Hibiscus syriacus üzerinden, Çorakçılar Parkı (Melikgazi), 20.VIII.2014; Erciyes evler Parkı (Talas), 06.VIII.2014; Platanus orientalis üzerinden, Şelale Parkı (Hacılar), 10.V.2014; Robinia pseudoacacia üzerinden, İncesu Parkı (İncesu), 10.V.2014; Rubus sp. üzerinden, Adnan Menderes Parkı (Melikgazi), 24.V.2014; Cemilbaba Parkı (Talas), 16.V.2015; Hisarcık Fatih Parkı (Melikgazi), 02.VI.2015; Hisarcık Illker Başbuğ Parkı (Melikgazi), 23.V.2015, 09.VI.2015; Salix sp. üzerinden, Adnan Menderes Parkı (Melikgazi), 24.V.2014.

Zoocoğrafi Dağılımı: Kozmopolit bir türdür (Nieto Nafria, 2017).

Türkiye'deki Dağııımı: Adana, Ağrı, Aksaray, Amasya, Ankara, Antalya, Artvin, Aydın, Balıkesir, Bartın, Bitlis, Bolu, Burdur, Bursa, Çanakkale, Çankırı, Denizli, Diyarbakır, Edirne, Elazığ, Erzurum, Eskişehir, Gaziantep, Giresun, Gümüşhane, Hatay, Isparta, İstanbul, İzmir, Kahramanmaraş, Kastamonu, Kayseri, Kırşehir, Konya, Manisa, Mardin, Mersin, Niğde, Sakarya, Samsun, Şanlıurfa, Trabzon, Uşak, Van, Yalova, Yozgat (Bodenheimer \& Swirski, 1957; Giray, 1974; Çanakçığlu, 1975; Düzgüneş et al., 1982; Toros et al., 2002; Tuatay, 1993; Akkaya \& Uygun, 1996; Toros et al., 1996; Çobanoğlu, 2000; Uygun et al., 2001; Ölmez Bayhan et al., 2003; Görür, 2004; Altay \& Uysal, 2005; Aslan \& Uygun, 2005; Ünal \& Özcan, 2005; Özdemir et al., 2006; Geneci \& Görür, 2007; Şahin, 2007; Çıraklı et al., 2008; Narmanlıoğlu \& Güçlü, 2008; Toper Kaygın et al., 2008; Eser et al., 2009; Güleç, 2011; Kuloğlu, 2011; Akyürek et al., 2012; Akyürek, 2013; Akyıldırım et al., 2014; Yıldırım \& Eroğlu, 2015; Kök et al., 2016).

\section{Aphis (Aphis) fabae Scopoli, 1763}

İncelenen materyal: Hedera helix üzerinden, Şehit Er Veysel Ekşi Parkı (Talas), 15.V.2015; Meydan (Melikgazi), 15.V.2015; Muhsin Yazıcıoğlu Parkı (Talas), 20.V.2015; Melikgazi Emniyet Müdürlüğü (Melikgazi), 22.V.2015; Meydan (Melikgazi), 27.V.2015; Philadelphus coronarius üzerinden, Cemilbaba Parkı (Talas), 13.V.2015; Yol kenarı (Hisarcık), 25.VI.2015; Viburnum opulus üzerinden, Şelale Parkı (Hacılar), 21.IV.2014; 04.VII.2015; Hisarcık Fatih Parkı (Melikgazi), 16.V.2015; 02.VI.2015; Erciyes Üniversitesi Kampüsü (Melikgazi), 17.V.2015; 20.V.2015; 24.V.2014; Meydan (Melikgazi), 20.V.2015; Göğüs Hastalıkları Hastanesi (Talas), 31.V.2015; Hisarcık Yol kenarı (Melikgazi), 23.VI.2015; 
03.VIII.2015; Hisarcık Meydan (Melikgazi), 20.VII.2015; 03.VIII.2015; Viburnum orientale üzerinden, Şelale Parkı (Hacılar), 10.V.2014; 13.V.2014; Kıranardı Meydan Cami (Melikgazi), 24.V.2014; Erciyes Üniversitesi Kampüsü (Melikgazi), 31.V.2014; 11.V.2015.

Zoocoğrafi Dağılımı: Avustralyan bölgesi hariç tüm dünya (Nieto Nafria, 2017).

Türkiye'deki Dağılımı: Adana, Aksaray, Amasya, Ankara, Antalya, Artvin, Aydın, Balıkesir, Bartın, Bolu, Burdur, Bursa, Çanakkale, Çankırı, Denizli, Diyarbakır, Edirne, Erzurum, Gaziantep, Giresun, Hatay, İstanbul, İzmir, Kahramanmaraş, Kastamonu, Kayseri, Kırklareli, Konya, Manisa, Mersin, Muğla, Niğde, Osmaniye, Rize, Sakarya, Samsun, Sinop, Şanlıurfa, Tekirdağ, Tokat, Trabzon, Van, Yalova, Zonguldak (İyriboz, 1937; Schimitschek, 1944; Bodenheimer \& Swirski, 1957; Tuatay \& Remaudiere, 1964; Çanakçıoğlu, 1967; Giray, 1974; Çanakçıŏlu,1967; Çanakçıoğlu, 1975; Düzgüneş et al., 1982; Zeren, 1989; Tuatay, 1993; Toros et al., 1996; Özdemir \& Toros, 1997; Uygun et al., 2001; Toros et al., 2002; Ölmez Bayhan et al., 2003; Altay \& Uysal, 2005; Aslan \& Uygun, 2005; Ayyıldız \& Atlıhan, 2006; Özdemir et al., 2006; Sönmezyıldız, 2006; Geneci \& Görür, 2007; Şahin, 2007; Çıraklı et al., 2008; Toper Kaygın et al., 2008; Toper Kaygın et al., 2009; Eser et al., 2009; Görür et al., 2009; Güleç, 2011; Kuloğlu, 2011; Akyürek, 2013; Akyıldırım et al., 2014; Kök et al., 2016).

\section{Aphis (Aphis) gossypii Glover, 1854}

Incelenen materyal: Hibiscus syriacus üzerinden, Çorakçılar Parkı (Melikgazi), 20.VIII.2014; Ligustrum vulgare üzerinden, Talas Erkek Yurdu (Talas), 08.VI.2014; Morus sp. üzerinden, Adnan Menderes Parkı (Talas), 24.V.2014; Philadelphus coronarius üzerinden, Cemilbaba Parkı (Talas), 28.V.2015; Rubus sp. üzerinden, Cemilbaba Parkı (Talas), 28.V.2015; Göğüs Hastalıkları Hastanesi (Talas), 31.V.2015; Hisarcık Fatih Parkı (Melikgazi), 02.VI.2015.

Zoocoğrafi Dağılımı: Kozmopolit bir türdür (Nieto Nafria, 2017).

Türkiye'deki Dağılımı: Adana, Aksaray, Amasya, Ankara, Antalya, Artvin, Aydın, Balıkesir, Bartın, Bolu, Burdur, Çanakkale, Denizli, Diyarbakır, Erzincan, Erzurum, Giresun, Hatay, Iğdır, İstanbul, İzmir, Kahramanmaraş, Kars, Kayseri, Konya, Mersin, Muğla, Niğde, Ordu, Sakarya, Samsun, Şanlıurfa, Trabzon, Van, Yalova (lyriboz, 1937; Düzgüneş et al., 1982; Tuatay, 1993; Toros et al., 1996; Toros et al., 2002; Ölmez Bayhan et al., 2003; Görür, 2004; Altay ve Uysal, 2005; Aslan \& Uygun, 2005; Ayyıldız \& Atlıhan, 2006; Özdemir et al., 2006; Sönmezyıldız, 2006; Geneci \& Görür, 2007; Şahin, 2007; Çıraklı et al., 2008; Toper Kaygın et al., 2008; Eser et al., 2009; Toper Kaygın et al., 2009; Güleç, 2011; Akyürek et al., 2012; Akyürek, 2013; Tuatay, 1993; Altay \& Uysal, 2005; Kuloğlu, 2011; Sangün \& Satar, 2012; Akyıldırım et al., 2014; Kök et al., 2016).

\section{Aphis (Aphis) pomi De Geer, 1773}

İncelenen materyal: Berberis thunbergii üzerinden, Erciyes Üniversitesi Kampüsü (Melikgazi), 13.IV.2014; Şelale Parkı (Hacılar), 10.V.2014; Adliye Parkı (İncesu) 10.V.2014; Cydonia sp. üzerinden, Fuar Parkı (Kocasinan), 17.V.2014; İnönü Parkı (Kocasinan), 17.V.2014; Halef hoca Mezarlığı (Talas), 31.V.2014; Spirea vanhouetti üzerinden, Şelale Parkı (Hacılar), 10.V.2014.

Zoocoğrafi Dağılımı: Afro-tropikal bölge hariç tüm dünya (Nieto Nafria, 2017).

Türkiye'deki Dağılımı: Adana, Amasya, Ankara, Antalya, Artvin, Bartın, Bitlis, Burdur, Bursa, Çanakkale, Diyarbakır, Edirne, Elazığ, Erzincan, Erzurum, Gaziantep, Giresun, Gümüşhane, Hatay, Iğdır, Isparta, İstanbul, İzmir, Kahramanmaraş, Kars, Kayseri, Kırklareli, Konya, Mersin, Nevşehir, Niğde, Ordu, Rize, Samsun, Şanlıurfa, Trabzon, Van (Bodenheimer \& Swirski, 1957; Düzgüneş \& Toros, 1978; Çanakçıŏlu, 1975; Erkin, 1983; Tuatay, 1993; Erol \& Yaşar, 1996; Toros et al., 1996; Güçlü et al., 1998; Toros et al., 2002; Ölmez Bayhan et al., 2003; Görür, 2004; Aslan \& Uygun, 2005; Çota, 2007; Şahin, 
2007; Daşçı \& Güçlü, 2008; Toper Kaygın et al., 2009; Ayaz \& Yücel, 2010; Güleç, 2011; Akyürek et al., 2012; Akyürek, 2013; Narmanlıoğlu, 2013; Akyıldırım et al., 2014; Kök et al., 2016).

\section{Hyalopterus pruni (Geoffroy, 1762)}

İncelenen materyal: Prunus cerasifera üzerinden Erciyes Üniversitesi Kampüsü (Melikgazi), 20.VI.2014; Üçgöz Köprü Parkı (İncesu), 21.VIII.2014.

Zoocoğrafi Dağııımı: Kozmopolit bir türdür (Nieto Nafria, 2017).

Türkiye'deki Dağılımı: Adana, Aksaray, Ankara, Antalya, Artvin, Bartın, Çanakkale, Denizli, Diyarbakır, Erzincan, Erzurum, Gaziantep, Hatay, Iğdır, Isparta, İstanbul, İzmir, Kahramanmaraş, Kars, Kayseri, Konya, Malatya, Mersin, Niğde, Samsun, Tekirdağ, Van (Düzgüneş \& Tuatay, 1956; Bodenheimer \& Swirski, 1957; Çanakçıoğlu, 1975; Düzgüneş et al., 1982; Toros et al., 1996; Güçlü et al., 1998; Uygun et al., 2001; Toros et al., 2002, Ölmez Bayhan et al., 2003; Öztürk et al., 2004; Aslan \& Karaca, 2005; Altay \& Uysal, 2005; Aslan \& Uygun, 2005; Özkan et al., 2005; Çota, 2007; Geneci \& Görür, 2007; Şahin, 2007; Çıraklı et al., 2008; Daşçı \& Güçlü, 2008; Narmanlıoğlu \& Güçlü, 2008; Toper Kaygın et al., 2008; Eser et al., 2009; Hazır \& Ulusoy, 2012; Akyürek, 2013; Narmanlıoğlu, 2013; Rakauskas et al., 2015; Kök et al., 2016).

\section{Rhopalosiphum nymphaeae (Linnaeus, 1761)}

İncelenen materyal: Pyrus elaeagrifolia üzerinden Kıranardı (Melikgazi), 24.V.2014.

Zoocoğrafi Dağılımı: Kozmopolit bir türdür (Nieto Nafria, 2017).

Türkiye'deki Dağılımı: Ankara, Antalya, Erzurum, İzmir, Kahramanmaraş, Mersin, Sakarya, Van (Bodenheimer \& Swirski, 1957; Tuatay ve Remaudiere, 1964; Erkin, 1983; Özdemir \& Toros, 1997; Güleç, 2011; Sangün \& Satar, 2012; Narmanlıoğlu, 2013; Rakauskas et al., 2015). Kayseri faunası için yeni kayıttır.

\section{Rhopalosiphum rufiabdominale (Sasaki, 1899)}

İncelenen materyal: Viburnum opulus üzerinden, Cumhuriyet meydanı (Melikgazi), 20.V.2015.

Zoocoğrafi Dağılımı: Tüm dünyada yaygınlık gösteren kozmopolit bir türdür (Nieto Nafria, 2017).

Türkiye'deki Dağılımı: Batı Anadolu Bölgesi (Önder et al., 2011). Kayseri faunası için yeni kayıttır.

\section{Tribe: Macrosiphini}

\section{Amphorophora (Amphorophora) rubi (Kaltenbach, 1843)}

İncelenen materyal: Rubus sp. üzerinden, Ş. P. Er Hatem Uzunoğlu Parkı (Talas), 03.V.2014; Şelale Parkı (Hacılar), 04.VIII.2014; Hisarcık-İlker Başbuğ Parkı (Melikgazi), 23.V.2015.

Zoocoğrafi Dağılımı: Avustralyan, Nearktik ve Palaearktik Bölge (Nieto Nafria, 2017). kayıttır.

Türkiye'deki Dağılımı: Ankara, Bitlis, Erzincan, Rize (Tuatay, 1993) Kayseri faunası için yeni 


\section{Aulacorthum (Aulacorthum) solani (Kaltenbach, 1843)}

İncelenen materyal: Viburnum orientale üzerinden, Şelale Parkı (Hacılar), 10.V.2014; Rubus sp. üzerinden, Ş. P. Er Hatem Uzunoğlu Parkı (Talas), 25.Vı.2014.

Zoocoğrafi Dağııımı: Kozmopolit bir türdür (Nieto Nafria, 2017).

Türkiye'deki Dağılımı: Adana, Antalya, Aydın, Diyarbakır, Çanakkale, Erzincan, Eskişehir, İstanbul, İzmir, Mersin, Niğde, Osmaniye, Samsun, Van (Tuatay, 1988; Toros et al., 2002; Ölmez Bayhan et al., 2003; Görür, 2004; Eser et al., 2009; Güleç, 2011; Akyürek et al., 2012; Sangün \& Satar, 2012; Akyürek, 2013; Kök et al., 2016).

\section{Brachycaudus (Brachycaudus) helichrysi (Kaltenbach, 1843)}

İncelenen materyal: Cydonia sp. üzerinden, İnönü Parkı (Kocasinan), 17.V.2014; Sorbus sp. üzerinden Erciyes Üniversitesi Kampüsü (Melikgazi), 31.V.2014; Rubus sp. üzerinden, Hisarcık-Fatih Parkı (Melikgazi), 02.VI.2015, 16.VI.2015.

Zoocoğrafi Dağılımı: Kozmopolit bir türdür (Nieto Nafria, 2017).

Türkiye'deki Dağılımı: Adana, Adıyaman, Aksaray, Ankara, Antalya, Artvin, Bartın, Burdur, Çankırı, Çanakkale, Denizli, Diyarbakır, Erzincan, Erzurum, Eskişehir, Gaziantep, Gümüşhane, Hatay, Isparta, İstanbul, İzmir, Kahramanmaraş, Kayseri, Mardin, Mersin, Niğde, Rize, Samsun, Sakarya, Siirt, Trabzon, Van (Tuatay \& Remaudiere, 1964; Tuatay et al., 1967; Tuatay et al., 1972; Giray, 1974; Düzgüneş et al., 1982; Tuatay, 1988; Güçlü et al., 1998; Toros et al., 2002; Ölmez Bayhan et al., 2003; Aslan Karaca, 2005; Aslan \& Uygun, 2005; Özdemir et al., 2006; Geneci \& Görür, 2007; Çota, 2007; Şahin, 2007; Çıraklı et al., 2008; Eser et al., 2009; Görür et al., 2009; Toper Kaygın et al., 2009; Akyürek, 2013; Narmanlıoğlu, 2013; Akyıldırım et al., 2014; Kök et al., 2016).

\section{Cavariella (Cavariella) aegopodii (Scopoli, 1763)}

Incelenen materyal: Salix sp. üzerinden, Muhsin Yazıcıoğlu Parkı (Talas), 27.V.2014.

Zoocoğrafi Dağılımı: Kozmopolit bir türdür (Nieto Nafria, 2017).

Türkiye'deki Dağıımı: Ankara, Antalya, Bartın, Burdur, Çanakkale, Çankırı, Erzurum, Eskişehir, İstanbul, İzmir, Van (Bodenheimer \& Swirski, 1957; Giray, 1974; Tuatay, 1988; Çota, 2007; Toper Kaygın et al., 2009; Güleç, 2011; Kök et al., 2016). Kayseri faunası için yeni kayıttır.

\section{Chaetosiphon (Pentatrichopus) tetrarhodum (Walker, 1849)}

İncelenen materyal: Rosa sp. üzerinde, Erciyes Üniversitesi Kampüsü (Melikgazi), 18.IV.2014, 08.VI.2014, 25.VI.2014; Bahçelievler (Talas), 21.IV.2014; Mimar Sinan Parkı (Kocasinan), 24.IV.2014; Büyükşehir Belediye Parkı (Melikgazi), 30.IV.2014; Talas Erkek Yurdu (Talas), 08.VI.2014; J. Komutanı Şevket Eygi Parkı (Talas), 08.Vı.2014; Karayolları 6. Bölge Müdürlüğü (Melikgazi), 20.VIII.2014; Hacılar Merkez (Hacılar), 25.VIII.2014; Hisarcık-Kayadibi Cami (Melikgazi), 27.VI.2015; Rosa canina üzerinde, Ali dağı Mesire alanı (Talas), 24.V.2014.

Zoocoğrafi Dağılımı: Kozmopolit bir türdür (Nieto Nafria, 2017).

Türkiye'deki Dağıımı: Ankara, Antalya, Burdur, Giresun, Isparta, Kayseri, Konya (Tuatay \& Remaudiere, 1964; Tuatay, 1988; Güleç, 2011; Akyürek et al., 2012). 


\section{Dysaphis (Dysaphis) crataegi (Kaltenbach, 1843)}

İncelenen materyal: Crataegus sp. üzerinde Kıranardı (Melikgazi), 24.V.2014; Şelale Parkı (Hacılar), 25.VIII.2014; Erciyes Üniversitesi Kampüsü (Melikgazi), 06.VIII.2014, 30.VIII.2014; Hisarcık-Yol kenarı (Melikgazi), 27.VI.2015.

Zoocoğrafi Dağılımı: Palaearktik ve Nearktik Bölge (Nieto Nafria, 2017).

Türkiye'deki Dağılımı: Ankara, Antalya, Isparta, İstanbul, Kastamonu, Konya, Van (Acatay, 1943; Çanakçıoğlu, 1975; Düzgüneş et al., 1982; Tuatay, 1990; Güleç, 2011). Kayseri faunası için yeni kayıttır.

\section{Liosomaphis berberidis (Fitch, 1851)}

İncelenen materyal: Berberis thunbergii üzerinde, Adliye Parkı (İncesu), 10.V.2014.

Zoocoğrafi Dağılımı: Avustralyan, Palaearktik ve Nearktik Bölge (Nieto Nafria, 2017).

Türkiye'deki Dağılımı: Ankara, Bitlis, Çanakkale, Çankırı, Giresun, Isparta, İstanbul, Konya (Tuatay \& Remaudière, 1964; Tuatay, 1990; Barjadze et al., 2014; Kök et al., 2016). Kayseri faunası için yeni kayııttır.

\section{Macrosiphum (Macrosiphum) euphorbiae (Thomas, 1878)}

İncelenen materyal: Berberis thunbergii üzerinden, Talas Erkek Yurdu (Talas), 08.VI.2014; Erciyes Üniversitesi Kampüsü (Melikgazi), 14.V.2015; Rubus sanctus üzerinden, Hisarcık Fatih Parkı (Melikgazi), 16.V.2015.

Zoocoğrafi Dağılımı: Kozmopolit bir türdür (Nieto Nafria, 2017).

Türkiye'deki Dağılımı: Adana, Aksaray, Amasya, Ankara, Antalya, Balıkesir, Bartın, Denizli, Edirne, Erzurum, Hatay, İstanbul, İzmir, Kayseri, Konya, Mersin, Niğde, Sakarya, Samsun (Tuatay \& Remaudiere, 1964; Tuatay, 1990; Çobanoğlu, 2000; Toros et al., 2002; Bayındır, 2003; Altay \& Uysal, 2005; Ayyıldız \& Atlıhan, 2006; Özdemir et al., 2006; Geneci \& Görür, 2007; Çota, 2007; Şahin, 2007; Çıraklı et al., 2008; Eser et al., 2009; Toper Kaygın et al., 2009; Güleç, 2011; Akyürek et al., 2012; Akyürek, 2013; Kök et al., 2016).

\section{Macrosiphum (Macrosiphum) rosae (Linnaeus, 1758)}

İncelenen materyal: Rosa sp. üzerinde, Erciyes Üniversitesi Kampüsü (Melikgazi), 14.IV.2014, 03.V.2014; Adliye Parkı (İncesu), 10.V.2014; Şelale Parkı (Hacılar), 13.V.2014; Şeh. Piy. Ast. Osman Y. Parkı (Talas), 15.V.2014.

Zoocoğrafi Dağılımı: Kozmopolit bir türdür (Nieto Nafria, 2017).

Türkiye'deki Dağıımı: Adana, Ankara, Antalya, Artvin, Bartın, Bolu, Burdur, Çanakkale, Çankırı, Denizli, Diyarbakır, Edirne, Giresun, Hatay, Isparta, İstanbul, İzmir, Kahramanmaraş, Kayseri, Konya, Mersin, Rize, Sakarya, Samsun, Trabzon, Van (Düzgüneş \& Tuatay, 1956; Bodenheimer \& Swirski, 1957; Tuatay et al., 1972; Giray, 1974; Çanakçıŏlu, 1975; Düzgüneş et al., 1982; Tuatay, 1990; Toros et al., 1996; Toros et al., 2002; Ölmez Bayhan et al., 2003; Altay, 2004; Aslan \& Uygun, 2005; Çota, 2007; Şahin, 2007; Çıraklı et al., 2008; Eser et al., 2009; Toper Kaygın et al., 2009; Güleç, 2011; Akyürek et al., 2012; Akyürek, 2013; Barjadze et al., 2014; Kök et. al., 2016). 


\section{Metopolophium (Metopolophium) dirhodum (Walker, 1849)}

İncelenen materyal: Rosa canina üzerinde, Hisarcık Fatih Parkı (Melikgazi), 23.V.2015.

Zoocoğrafi Dağılımı: Kozmopolit bir türdür (Nieto Nafria, 2017).

Türkiye'deki Dağılımı: Adana, Bursa, Ankara, Bartın, Bitlis, Çankırı, Elazığ, Erzurum, Isparta, Kahramanmaraş, Konya, Mersin, Niğde, Sakarya (Bodenheimer \& Swirski, 1957; Toros et al., 2002; Aslan \& Uygun, 2005; Çota, 2007; Yıldırım \& Eroğlu, 2015). Kayseri faunası için yeni kayıttır.

\section{Myzaphis rosarum (Kaltenbach, 1843)}

İncelenen materyal: Rosa sp. üzerinde, Erciyes Üniversitesi Kampüsü (Melikgazi), 14.IV.2014, 16.IV.2014, 18.IV.2014, 30.IV.2014, 08.VI.2014, 25.VI.2014, 11.V.2015; Büyükşehir Belediye Parkı (Melikgazi), 30.IV.2014; Karayolları 6. Bölge Müdürlüğü (Melikgazi), 20.VIII.2014; Merkez (Hacılar), 25.VIII.2014; Şaşaoğlu Cami (Hacılar), 04.VIII.2014; Cemilbaba Parkı (Talas), 28.V.2015; Hisarcık İlker Başbuğ Parkı (Melikgazi), 25.VI.2015; Hisarcık Kayadibi Cami (Melikgazi), 27.VI.2015; Hisarcık Meydan (Melikgazi), 20.VII.2015.

Zoocoğrafi Dağılımı: Kozmopolit bir türdür (Nieto Nafria, 2017).

Türkiye'deki Dağılımı: Ankara, Bolu, Erzurum, Kayseri, Niğde (Düzgüneş \& Tuatay, 1956; Tuatay \& Remaudiere, 1964; Kavaz, 2006; Şahin, 2007).

\section{Myzus (Myzus) cerasi (Fabricius, 1775)}

İncelenen materyal: Prunus mahaleb üzerinde, Erciyes Üniversitesi Kampüsü (Melikgazi) 01.VI.2015. P. mahaleb Türkiye'de M. cerasi için yeni konukçu bitkidir.

Zoocoğrafi Dağılımı: Avustralyan, Palaearktik, Nearktik ve Oryantal Bölge (Nieto Nafria, 2017).

Türkiye'deki Dağılımı: Adana, Ankara, Antalya, Artvin, Bartın, Bursa, Çanakkale, Diyarbakır, Elazığ, Erzurum, Gümüşhane, Iğdır, Isparta, İstanbul, İzmir, Kahramanmaraş, Kars, Kayseri, Kocaeli, Konya, Mardin, Mersin, Niğde, Rize, Samsun, Tekirdağ, Trabzon, Van (Bodenheimer \& Swirski, 1957; Düzgüneş et al., 1982; Tuatay, 1991; Toros et al., 1996; Güçlü et al., 1998; Uygun et al., 2001; Toros et al., 2002; Ölmez Bayhan et al., 2003; Altay, 2004; Çınar et al., 2004; Aslan \& Karaca, 2005; Aslan \& Uygun, 2005; Narmanlıoğlu, 2006; Çota, 2007; Şahin, 2007; Daşçı \& Güçlü, 2008; Narmanlıoğlu \& Güçlü, 2008; Güleç, 2011; Akyürek et al., 2012; Akyürek, 2013; Narmanlıoğlu, 2013; Akyıldırım et al., 2014; Barjadze et al., 2014; Kök et al., 2016).

\section{Myzus (Nectarosiphon) persicae Sulzer, 1776}

İncelenen materyal: Cercis siliquastrum üzerinde, Adliye Parkı (İncesu), 10.V.2014; Hedera helix üzerinde, Melikgazi Emniyet Müdürlüğü (Melikgazi), 11.V.2015; Meydan (Talas), 15.V.2015; Robinia pseudoacacia üzerinde, Üçgöz Köprü Parkı (İncesu), 10.V.2014; Muhsin Yazıcıoğlu Parkı (Talas), 13.V.2014.

Zoocoğrafi Dağılımı: Kozmopolit bir türdür (Nieto Nafria, 2017).

Türkiye'deki Dağııımı: Adana, Amasya, Ankara, Antalya, Artvin, Aydın, Balıkesir, Bartın, Bolu, Bursa, Çanakkale, Diyarbakır, Edirne, Erzurum, Eskişehir, Giresun, Hatay, Iğdır, Isparta, İstanbul, İzmir, Kahramanmaraş, Kayseri, Kırklareli, Kocaeli, Konya, Manisa, Malatya, Mersin, Muş, Niğde, Rize, Sakarya, Samsun, Sinop, Şırnak, Tekirdağ, Tokat, Trabzon, Tunceli, Uşak, Van (Bodenheimer \& Swirski 1957; Düzgüneş et al., 1982; Erkin, 1983; Tuatay, 1991; Önuçar \& Ulu, 1992; Kıran, 1994; Özbek et al., 
1996; Toros et al., 1996; Güçlü et al., 1998; Çobanoğlu, 2000; Toros et al., 2002; Ölmez Bayhan et al., 2003; Öztürk et al., 2004; Aslan \& Karaca, 2005; Aslan \& Uygun, 2005; Ayyıldız \& Atlıhan, 2006; Sönmezyıldız, 2006; Şahin, 2007; Eser et al., 2009; Görür et al., 2009; Toper Kaygın et al., 2009; Güleç, 2011; Akyürek et al., 2012; Sangün \& Satar, 2012; Akyürek, 2013; Narmanlıoğlu, 2013; Rakauskas et al., 2015; Kök et al., 2016).

\section{Altfamilya: Callaphidinae}

\section{Tribe: Panaphini}

\section{Eucallipterus tilia (Linnaeaus, 1758)}

İncelenen materyal: Tilia sp. üzerinde, İncesu parkı (İncesu), 10.V.2014.

Zoocoğrafi Dağılımı: Avustralyan, Nearktik, Neotropikal ve Palaearktik Bölge (Nieto Nafria, 2017).

Türkiye'deki Dağılımı: Ankara, Bartın, Isparta, İstanbul, İzmir, Kastamonu, Niğde, Rize, Samsun, Trabzon, Van (Tuatay \& Remaudiere, 1964; Çanakçıŏlu, 1966, Çanakçıoğlu, 1967; Tuatay et al., 1967; Çanakçıŏlu, 1975; Ünal \& Özcan, 2005; Toper Kaygın et al., 2008; Eser et al., 2009; Görür et al., 2009; Atıhan et al., 2011; Akyürek, 2013; Demirözer et al., 2015; Kök et al., 2016). Kayseri faunası için yeni kayittır.

\section{Myzocallis (Myzocallis) coryli (Goetze,1778)}

İncelenen materyal: Corylus avellana üzerinde, İnönü Parkı (Kocasinan), 17.V.2014; Erciyes Üniversitesi Kampüsü (Melikgazi), 14.V.2015, 26.V.2015.

Zoocoğrafi Dağılımı: Avustralyan, Nearktik, Neotropikal, Palaearktik ve Oryantal Bölge (Nieto Nafria, 2017).

Türkiye'deki Dağılımı: Ankara, Diyarbakır, Doğu Karadeniz Bölgesi, Isparta, İstanbul, Niğde, Trabzon (Fahringer, 1922; Bodenheimer \& Swirski, 1957; Çanakçığlu, 1966; Çanakçığlu, 1967; Tuatay et al., 1967; Ural et al., 1973; Çanakçıoğlu, 1975; Düzgüneş et al., 1982; Ölmez Bayhan et al., 2003; Aslan \& Karaca, 2005; Kocadal, 2006; Görür et al., 2009; Akyürek, 2013). Kayseri faunası için yeni kayittır.

\section{Altfamilya: Eriosomatinae}

\section{Tribus: Eriosomatini}

\section{Eriosoma lanigerum (Hausmann, 1802)}

İncelenen materyal: Malus sp. üzerinde, Erciyes Evler Parkı (Talas), 06.VIII.2014.

Zoocoğrafi Dağılımı: Kozmopolit bir türdür (Nieto Nafria, 2017).

Türkiye'deki Dağıımı: Adana, Afyon, Ankara, Antalya, Artvin, Balıkesir, Bursa, Çanakkale, Çorum, Diyarbakır, Elazığ, Erzincan, Erzurum, Eskişehir, Hatay, Isparta, İstanbul, Kahramanmaraş, Kastamonu, Kayseri, Kırşehir, Kocaeli, Konya, Kütahya, Malatya, Mardin, Mersin, Niğde, Samsun, Trabzon (Schmitschek, 1944; Bodenheimer \& Swirski, 1957; Düzgüneş \& Toros, 1978; Düzgüneş et al., 1982; Güçlü et al., 1998; Toros et al., 2002; Ölmez Bayhan et al., 2003; Aslan \& Karaca, 2005; Aslan \& Uygun, 2005; Bozbek et al., 2009; Görür et al., 2009; Ayaz \& Yücel, 2010; Güleç, 2011; Akyürek, 2013; Narmanlıoğlu, 2013). 


\section{Altfamilya: Lachninae}

\section{Tribe: Eulachnini}

\section{Cinara (Cinara) cedri Mimeur, 1936}

İncelenen materyal: Cedrus sp. üzerinde, Şelale Parkı (Hacılar), 10.05.2014; Erciyes Üniversitesi Kampüsü (Melikgazi), 25.06.2014, 20.08.2014; Hacılar Müftülüğü (Hacılar), 25.08.2014; Feyyaz Mercan İlkokulu (Hacılar), 04.08.2014; Feyyaz Mercan Cami (Hacılar), 07.08.2014.

Zoocoğrafi Dağılımı: Neotropikal ve Palaearktik Bölge (Nieto Nafria, 2017).

Türkiye'deki Dağılımı: Afyon, Ankara, Antalya, Artvin, Bartın, Burdur, Eskişehir, Gaziantep, Hatay, İstanbul, Kahramanmaraş, Kastamonu, Kayseri, Konya, Samsun, Tekirdağ (Tuatay \& Remaudiere, 1964; Çanakçıŏlu, 1966; Çanakçıŏlu, 1967; Tuatay et al., 1967; Çanakçıoğlu, 1975; Düzgüneş et al., 1982; Tuatay, 1999; Toros et al., 2002; Toper Kaygın \& Çanakçığlu, 2003; Altay \& Uysal, 2005; Aslan \& Uygun, 2005; Ünal \& Özcan, 2005; Şahin, 2007; Toper Kaygın et al., 2008; Görür et al., 2009; Akyürek et al., 2012; Akyürek, 2013).

\section{Cinara (Cubressobium) cupressi (Buckton, 1881)}

İncelenen materyal: Cupressus sp. üzerinde, Merkez (Hacılar), 25.VIII.2014; Thuja sp. üzerinde, İncesu Parkı (İncesu), 10.V.2014; Şelale Parkı (Hacılar), 08.VIII.2014; Erciyes Üniversitesi Kampüsü (Melikgazi), 30.VIII.2014.

Zoocoğrafi Dağılımı: Kozmopolit bir türdür (Nieto Nafria, 2017).

Türkiye'deki Dağılımı: Antalya, Bartın, Kahramanmaraş, Kastamonu, Konya, Antalya, Samsun (Aslan \& Uygun, 2005; Atalay \& Uysal, 2005; Ünal \& Özcan, 2005; Toper Kaygın et al., 2008; Güleç, 2011; Akyürek, 2013). Kayseri faunası için yeni kayıttır.

\section{Eulachnus rileyi (Williams, 1911)} 10.V.2014.

İncelenen materyal: Pinus sp. üzerinde, Şelale Parkı (Hacılar), 10.V.2014; Adliye Parkı (İncesu),

Zoocoğrafi Dağılımı: Afro-Tropikal, Nearktik, Neotropikal, Palaearktik ve Oryantal Bölge (Nieto Nafria, 2017).

Türkiye'deki Dağılımı: Ankara, Antalya, Bartın, Bolu, Çankırı, Edirne, Erzurum, İstanbul, Kastamonu, Kayseri, Konya, Mersin, Rize, Trabzon (Tuatay \& Remaudiere, 1964; Tuatay, 1993; Tuatay, 1999; Çobanoğlu, 2000; Toros et al., 2002; Altay \& Uysal, 2005; Ünal \& Özcan, 2005; Kavaz, 2006; Sönmezyıldız, 2006; Şahin, 2007; Güleç, 2011; Yıldııım \& Eroğlu, 2015).

\section{Lachnus roboris (Linnaeus, 1758)}

İncelenen materyal: Quercus sp. üzerinde, Meydan (Talas), 12.V.2014; Bahçelievler (Talas), 15.V.2014; Erciyes Üniversitesi Kampüsü (Melikgazi), 06.VIII.2014.

Zoocoğrafi Dağılımı: Nearktik, Palaearktik ve Oryantal Bölge (Nieto Nafria, 2017).

Türkiye'deki Dağılımı: Ankara, Artvin, Bitlis, Diyarbakır, Edirne, Erzincan, İstanbul, Kahramanmaraş, Mardin, Rize, Samsun, Trabzon (Fahringer, 1922; Schimitschek, 1944; Düzgüneş \& Tuatay, 1956; Bodenheimer \& Swirski, 1957; Çanakçıoğlu, 1966; Çanakçıoğlu, 1967; Çanakçıoğlu, 1975; 
Tuatay, 1999; Ölmez Bayhan et al., 2003; Aslan \& Uygun, 2005; Görür et al., 2009; Akyürek et al., 2012; Akyürek, 2013). Kayseri faunası için yeni kayıttır.

\section{Altfamilya: Mindarinae}

\section{Mindarus abietinus Koch, 1857}

İncelenen materyal: Cedrus sp. üzerinde, Feyyaz Mercan İlkokulu (Hacılar), 30.VIII.2014; Şaşaoğlu Cami (Hacılar), 05.VIII.2014; Feyyaz Mercan Cami (Hacılar), 05.VIII.2014.

Zoocoğrafi Dağııımı: Palaearktik, Nearktik ve Oryantal Bölgede (Nieto Nafria, 2017).

Türkiye'deki Dağılımı: Ankara, Artvin, Bolu, Burdur, Bursa, Çankırı, Giresun, İstanbul, Konya (Schimitschek, 1944; Düzgüneş \& Tuatay, 1956; Bodenheimer \& Swirski, 1957; Tuatay \& Remaudiere, 1964; Çanakçıoğlu, 1966; Tuatay, 1988). Kayseri faunası için yeni kayıttır.

\section{Altfamilya: Pterocommatinae}

\section{Pterocomma pilosum Buckton, 1879}

İncelenen materyal: Salix sp. üzerinde, Adnan Menderes Parkı (Melikgazi), 24.V.2014.

Zoocoğrafi Dağılımı: Nearktik, Palaearktik ve Oryantal Bölge (Nieto Nafria, 2017).

Türkiye'deki Dağılımı: Ankara, Isparta, Kahramanmaraş (Çanakçıŏlu, 1975; Aslan \& Uygun, 2005). Kayseri faunası için yeni kayıttır.

Türkiye, bölgelere göre değişen coğrafi ve iklim özellikleri nedeniyle oldukça zengin biyoçeşitliliğe sahip olduğu düşünülen bir ülkedir. Bununla birlikte, ülkemizde birçok canlı grubunun varlığı ve yaygınlığını belirlemek için yapılan çalışmaların sayısı oldukça sınırlıdır. Canlı çeşitliliği bazen aynı bölge içerisindeki lokal alanlar arasında bile farklılıklar gösterebilmektedir. Türkiye'de şu ana kadar 541 yaprakbiti türü (Görür et al., 2017) tespit edilmesine rağmen, farklı alanlarda yapılacak yeni çalışmalarla bu sayının çok daha fazla artması beklenmektedir. Bununla birlikte, daha önce tespit edilen türlerin dağıımının bilinmesi de çok önemli bir konudur. Burada, coğrafi ve iklim özellikleri ile birlikte konukçu bitki çeşitliliğinin de belirleyici bir rolü bulunmaktadır. Kayseri ili merkez ilçeleri, park ve bahçelerdeki bitki çeşitliliği yönünden oldukça zayıf bir bitki örtüsüne sahiptir. Buna karşın, yapılan bu çalışmada çalı ve odunsu formlara sahip park ve süs bitkileri üzerinde 30 yaprakbiti türü tespit edilmiştir. Yapılan literatür taramasında bu türlerden 14 tanesinin (Amphorophora (Amphorophora) rubi, Anoecia (Anoecia) corni, Cavariella (Cavariella) aegopodii, Cinara (Cubressobium) cubressi, Dysaphis (Dysaphis) crataegi, Eucallipterus tiliae, Lachnus roboris, Liosomaphis berberidis, Metopolophium (Metopolophium) dirhodum, Mindarus abietinus, Myzocallis (Myzocallis) coryli, Pterocomma pilosum, Rhopalosiphum nymphaeae ve Rhopalosiphum rufiabdominale) Kayseri ilinden kaydına rastlanılmadığı için, bu türler Kayseri ili için yeni kayıt olarak bildirilmiştir. Ayrıca, $P$. mahaleb Türkiye'de $M$. cerasi için yeni konukçu bitki olarak tespit edilmiştir. Süs bitkileri üzerinde $A$. craccivora, $A$. fabae, $A$. Gossypii ve $A$. pominin güller üzerinde ise Macrosiphum rosae ve Myzaphis rosarum'un yaygın türler olduğu belirlenmiştir. İleride yapılacak farklı alan ve konukçu bitki gruplarını da içeren, daha geniş çaplı çalışmalarla Kayseri yaprakbiti faunasına ait tür sayısının daha da artabileceği düşünülmektedir.

\section{Teşekkür}

Yaprakbitlerinin teşhisini yapan Dr. Işı Özdemir (Zirai Mücadele Merkez Araştırma Enstitüsü Müdürlüğü, Ankara)'e ve Konukçu bitkilerin teşhisini yapan Prof. Dr. Cem VURAL (Erciyes Üniversitesi, Fen Fakültesi, Biyoloji Bölümü, Kayseri)'a ve bu çalışmayı FYL-2014-5341 numaralı proje ile destekleyen Erciyes Üniversitesi Bilimsel Araştırma Projeleri Birimi'ne içtenlikle teşekkür ederiz. 


\section{Yararlanılan Kaynaklar}

Acatay, A., 1943. İstanbul Çevresi ve Bilhassa Belgrad Ormanındaki Zararlı Orman Böcekleri, Mücadeleleri ve İşletme Üzerine Tesirleri. T.C. Ziraat Vekaleti Yüksek Ziraat Enstitüsü Çalışmaları, Sayı:143, 163 s.

Akkaya, A \& N. Uygun, 1996. "Diyarbakır ve Şanlıurfa illeri yazlık sebze ekosistemindeki böcek faunası, s. 423-431". Türkiye 3. Entomoloji Kongresi (24-28 Eylül, Ankara) Bildirileri, 716 s.

Akyıldırım, H., Ö. Şenol, G. Görür, N. Aktaç, \& E. Demirtaş, 2014. Determined aphid and ant associations from Trabzon, Rize and Artvin provinces of the Turkey. Journal of the Entomological Research Society, 16 (2): 29 37.

Akyürek, B., 2013. Samsun İli Aphididae (Hemiptera: Aphidoidea) Familyası Türlerinin Taksonomik Yönden İncelenmesi. Ondokuz Mayıs Üniversitesi, Fen Bilimleri Enstitüsü, Doktora Tezi, Samsun, 378 s.

Akyürek, B., Ü. Zeybekoğlu \& G. Görür, 2012. Ondokuz Mayıs Üniversitesi Kurupelit Yerleşkesi (Samsun)'nin yaprakbiti (Hemiptera: Aphididae) türleri ve konukçu bitkileri. Türkiye Entomoloji Bülteni, 2 (2): 91-108.

Altay, H. \& M. Uysal, 2005. Selçuk Üniversitesi Alaeddin Keykubat Kampüs Alanında bulunan yaprakbitleri (Homoptera: Aphidoidea) türleri. Selçuk Üniversitesi Ziraat Fakültesi Dergisi, 19 (37): 92-99.

Aslan, B. \& I. Karaca, 2005. Fruit tree aphids and their natural enemies in Isparta Region, Turkey. Journal of Pest Science, 78: 227-229.

Aslan, M.M. \& N. Uygun, 2005. Aphids (Homoptera: Aphididae) of Kahramanmaraş province, Turkey. Turkish Journal of Zoology, 29: 201-209.

Atlıhan, R., M.S. Özgökçe, M.B. Kaydan, İ. Kasap, N. Kılınçer, S. Kıyak \& E. Polat, 2011.Van gölü havzası ceviz ağaçlarındaki böcek faunası. Türkiye Entomoloji Dergisi, 35 (2): 349-360.

Ayaz, T. \& A. Yücel, 2010. Elazığ İli Elma Alanlarında Bulunan Zararlı ve Yararlı Arthropod Türlerinin Belirlenmesi Üzerine Araştırmalar. Harran Üniversitesi Ziraat Fakültesi Dergisi, 14(1): 9-16.

Ayyıldız, Y. \& R. Atlıhan, 2006. Balıkesir ili sebze alanlarında görülen yaprakbiti türleri ve doğal düşmanları. Yüzüncü Yıl Üniversitesi Ziraat Fakültesi Tarım Bilimleri Dergisi, 16 (1): 1-5.

Barjadze, S., G. Japoshvili, İ. Karaca \& I. Özdemir, 2014. Aphids (Hemiptera: Aphidoidea) of Gölcük Natural Park (Isparta Province, Turkey). Munis Entomology \& Zoology Journal, 9 (1): 206-213.

Bayındır, N., 2003. Niğde İli ve Çevresinde Sebzelerde Zararlı Olan Afit (Insecta: Homoptera: Aphidoidea) Türlerinin Belirlenmesi. Niğde Üniversitesi Fen Bilimleri Enstitüsü Biyoloji Anabilim Dalı, Yüksek Lisans Tezi Niğde, 67s.

Blackman, R. L. \& V. F. Eastop, 2017. Aphids on the World's Plants an Online Identification and Information Guide, (Web sayfası: http://www.aphidsonworldsplants.info) (Erişim tarihi: Kasım 2017).

Bodenheimer, F. S. \& E. Swirski, 1957. The Aphidoidea of the Middle East. Weizmann Science Press of Israel, Jerusalem, 378 pp.

Bozbek, Ö., Y. Kütük, H. Alıcı, İ.F. Çakırbay \& A. Canbay, 2009. Erzincan'da elma pamuklubiti (Eriosoma lanigerum (Hausmann) (Hemiptera: Pemphigidae)'nin Yayılışı, Yoğunluğu, Parazitoit ve Predatörlerinin Tespiti. III. Bitki Koruma Kongresi (15-18 Temmuz Van) Bildirileri. s.375.

Çanakçığlu, H., 1966. Türkiye'de orman ağaçlarına arız olan bitki bitleri (Aphidoidea) üzerinde araştırmalar. İstanbul Üniversitesi Orman Fakültesi Dergisi, 16 (2): 131-190.

Çanakçıoğlu, H., 1967. Türkiye'de Orman Ağaçlarına Arız Olan Yaprakbitleri (Aphidoidea) Üzerine Araştırmalar. T.C. Tarım Bakanlığı, Orman Gn. Md. Yayınları Sıra No: 466, Seri No: 22. Ankara, 151 s. 
Çanakçıoğlu, H., 1975. The Aphidoidea of Turkey. İstanbul Üniversitesi Orman Fakültesi Yayınları. Yayın No: 189, 309 s.

Çınar, M., I. Çimen \& H. Bolu, 2004. Elazığ ve Mardin illeri kiraz ağaçlarında zararlı olan türler, doğal düşmanları ve önemlileri üzerinde gözlemler. Türkiye Entomoloji Dergisi, 28 (3): 213-220.

Çıraklı, A., G. Görür \& M. Işık, 2008. Denizli il merkezinde belirlenen afit (Hemiptera: Aphididae) türleri. Selçuk Üniversitesi Ziraat Fakültesi Dergisi, 22 (44): 12-18.

Çobanoğlu, S., 2000. Aphididae (Homoptera) species of edirne province (Thrace part of Turkey). Entomologist's Monthly Magazine, 45-52.

Çota, F., 2007. Bartın Yöresi Aphidoidae Türleri Üzerine Araştırmalar. Zonguldak Karaelmas Üniversitesi Fen Bilimleri Enstitüsü Orman Mühendisliği Anabilim Dalı, Yüksek Lisans Tezi, Zonguldak, 120s.

Daşçı, E. \& Ş. Güçlü, 2008. Iğdır ovasında meyve ağaçlarında bulunan yaprakbiti türleri (Homoptera: Aphididae) ve doğal düşmanları. Atatürk Üniversitesi Ziraat Fakültesi Dergisi, 39 (1): 71-73.

Demirözer, O., A. Uzun \& D. Şenal, 2015. Isparta il merkezinde bulunan ıhlamur ağaçları üzerinde saptanan trips ve yaprakbiti türleri. Türkiye Entomoloji Bülteni, 5 (1): 21-28.

Düzgünes, Z. \& N. Tuatay, 1956. Türkiye Aphid'leri. Ankara Ziraat Mücadele Enstitüsü Müdürlüğü, Sayı: 4: 1-63.

Düzgüneş, Z. \& S. Toros, 1978. Ankara İli ve Çevresinde Elma Ağaçlarında Bulunan Yaprakbiti Türleri ve Kısa Biyolojileri Üzerinde Araştırmalar. Türkiye Bitki Koruma Dergisi, 2 (3):151-175.

Düzgüneş, Z., S. Toros, N. Kılınçer \& B. Kovancı, 1982. Ankara Illinde Bulunan Aphidoidea Türlerinin Parazit ve Predatörlerinin Tespiti. Tarım ve Orman Bakanlığı, Zirai Mücadele ve Zirai Karantina Genel Müdürlüğü Yayınları, $251 \mathrm{~s}$.

Erkin, E., 1983. Investigations on the hosts distribution and efficiency on the natural enemies of the family Aphididae (Homoptera) harmful to pome and stone fruit tress in İmir province of aegean region. Türkiye Bitki Koruma Dergisi, 7 (1): 29-49.

Erol, T. \& B. Yaşar, 1996. Van ili elma bahçelerinde bulunan zararlı türler ile doğal düşmanları. Türkiye Entomoloji Dergisi, 20 (4): 281-293.

Eser, S. I., G. Görür, İ. Tepecik \& H. Akyıldırım, 2009. Aphid (Hemiptera: Aphidoidea) species of the Urla district of İzmir region. Journal of Applied Biological Sciences, 3 (1): 99-102.

Fahringer, J., 1922. Eine Rhynchotenausbeute aus der Türkei, Kleinasien und den benachbarten Gebieten. Konovia, 1: 296-307.

Geneci, E. \& G. Görür, 2007. Aphid (Homoptera: Aphididae) species of the Central Aksaray. International Journal of Natural of Engineering Sciences, 1: 19-21.

Giray, H., 1974. İzmir ili çevresinde Aphididae (Homoptera) familyası türlerine ait ilk liste ile bunların konukçu ve zarar şekilleri hakkında notlar. Ege Üniversitesi Ziraat Fakültesi Dergisi, 11 (1): 39-69.

Görür, G., 2004. Aphid (Homoptera : Aphididae) species on pome fruit trees in Nigde Province of Turkey. Turkiye Entomoloji Dergisi, 28 (1): 21-26.

Görür, G., Ü. Zeybekoğlu, B. Akyürek, M. Işık \& H. Akyıldıım, 2009. Trabzon, Rize ve Artvin Illlerinin Afit (Homoptera: Aphididae) Faunasının Belirlenmesi. Tübitak Projesi Nihai Raporu, Proje No: 107T450, 233 s.

Görür, G., O. Şenol, G. Gezici, H. Akyildirim Begen \& D. Parmaksiz, 2017. New aphid (Hemiptera: Aphidoidea) records from South Eastern Parts of Turkey. Journal of Insect Biodiversity and Systematics, 3 (3), 257-264. 
Güçlü, Ş., R. Hayat, H. Özbek, Ö. Çalmaşur \& S. Pekel, 1998. Artvin, Erzincan, Erzurum, Kars ve Iğdır illerinde meyve yetiştiriciliğinin entomolojik sorunları ve çözüm önerileri. Doğu Anadolu Tarım Kongresi (14-18 Eylül, Erzurum) Bildirileri, 24-35.

Güleç, G., 2011. Antalya Şehri Park Alanlarında Aphidoidea (Hemiptera) Türlerinin Saptanması ve Doğal Düşmanlarının Belirlenmesi. Ankara Üniversitesi, Fen Bilimleri Enstitüsü, Doktora Tezi, Ankara, 325 s.

Hazır, A. \& M.R. Ulusoy, 2012. Adana, Mersin illeri şeftali ve nektarin alanlarında saptanan zararlar ile predatör ve parazitoit türler. Türkiye Biyolojik Mücadele Dergisi, 3 (2): 157-168.

İyriboz, N., 1937. Pamuk Hastalıkları. Ankara Ziraat Vekaleti Neşriyatı, U. S. 237, Pamuk Bürosu S. 1. X+ 85 s.

Kavaz, H., 2006. Erzurum Atatürk Üniversitesi Kampüsünde Ağaç ve Çalı Formundaki Bitkilerde Bulunan Afit (Homoptera; Aphididae) Türleri ve Doğal Düşmanları. Fen Bilimleri Enstitüsü, Yüksek Lisans Tezi, Erzurum, $54 \mathrm{~s}$.

Kıran, E., 1994. Güneydoğu Anadolu Bölgesi hububat ekiliş alanlarında görülen yaprakbiti türleri ve doğal düşmanları üzerinde çalışmalar. 3. Biyolojik Mücadele Kongresi Bildirileri, Ankara, s.29-37.

Kocadal, E., 2006. Kuzey Kıbrıs Türk Cumhuriyeti'ndeki Aphidoidea (Homoptera) türleri, bunların konukçuları, parazitoit ve predatörlerinin belirlenmesi. Çukurova Üniversitesi Fen Bilimleri Enstitüsü Bitki Koruma Anabilim Dalı, Yüksek Lisans Tezi, Adana, 82s.

Kök, Ş., I. Kasap \& I. Özdemir, 2016. Aphid (Hemiptera: Aphididae) species determined in Çanakkale Province with a new record for the aphid fauna of Turkey. Türkiye Entomoloji Dergisi, 40 (4): 397-412.

Kuloğlu, İ., 2011. Yalova İlinde Bazı Süs Bitkilerinde Görülen Aphidoidea (Homoptera) Türleri Üzerine Araştırmalar. Namık Kemal Üniversitesi Fen Bilimleri Enstitüsü, Yüksek Lisans Tezi, Tekirdağ, 58 s.

Narmanlıoğlu, H. K. \& Ş. Güçlü, 2008. İspir (Erzurum) ilçesinde meyve ağaçlarında bulunan yaprakbiti türleri (Homoptera: Aphididae) ve doğal düşmanları. Atatürk Üniversitesi Ziraat Fakültesi Dergisi, 39 (2), 225-229.

Narmanlıoğlu, H.K., 2013. Yukarı Çoruh Vadisinde Yetiştirilen Ilıman İklim Meyvelerindeki Aphididae (Hemiptera) Türleri Ve Bunların Doğal Düşmanları. Atatürk Üniversitesi, Fen Bilimleri Enstitüsü, Doktora Tezi, Erzurum, $198 \mathrm{~s}$.

Nieto Nafria, J. M., 2017. Fauna Europaea: Hemiptera: Aphidoidea. Fauna Europaea version 2.6, (Web page: http://www.faunaeur.org) (Date accessed: Nov. 2017)

Rakauskas, R., M.M. Aslan, A.A. Işıkber, A. Zaremba, \& R. Bernotiene, 2015. Contribution to the knowledge of the orchard Aphid (Hemiptera: Aphididae) fauna of İstanbul and Kahramanmaraş. Kahramanmaraş Sütçü İmam Üniversitesi Doğa Bilimleri Dergisi, 18 (1): 13-16.

Sangün, O. \& S. Satar, 2012. Aphids (Hemiptera: Aphididae) on lettuce in the Eastern Mediterranean Region of Turkey: Incidence, population fluctuations, and flight activities. Türkiye Entomoloji Dergisi, 36 (4): 443-454.

Ölmez Bayhan, S., M. R. Ulusoy \& S. Toros, 2003. Determination of Aphididae (Homoptera) fauna of Diyarbakir province of Turkey. Türkiye Entomoloji Dergisi, 27 (4): 253-268.

Önder F., S. Tezcan, Y. Karsavuran \& Ü. Zeybekoğlu, 2011. Cicadomorpha, Fulgoromorpha and Sternorrhyncha (Insecta: Hemiptera) Catalogue of Turkey, Meta Basım Matbaacılık Hizmetleri, İzmir. 140 pp.

Önuçar, A. \& O. Ulu, 1992. Ege bölgesi meyve fidanlıklarındaki zararlılar üzerinde faunistik çalışmalar. Bitki Koruma Bülteni, 33 (1-2): 23-37.

Özbek, H., Ş. Güçlü \& R. Hayat, 1996. Investigations on the Phytophagous and Predator Insect Species on StoneFruits in North-East Agricultural Region of Turkey. Turkish Journal of Agriculture and Forestry, (20): 267-282. 
Özdemir, I. \& S. Toros, 1997. Ankara Parklarında Mevsimlik Süs Bitkilerinde Zararlı Aphidoidea (Hom.) Türleri. Türkiye Entomoloji Dergisi 21 (4):283-298.

Özdemir, I., S. Toros, A. N. Kılınçer \& M. O. Gürkan, 2006. A survey of Aphididae (Homoptera) on wild plants in Ankara, Turkey. Ekoloji, 15 (58): 38-41.

Özkan, C., O. Gürkan \& Ö. Hancıoğlu, 2005. Çubuk (Ankara) ilçesi vişne ağaçlarında zararlı olan türler, doğal düşmanları ve önemlileri üzerinde gözlemler. Tarım Bilimleri Dergisi, 11 (1): 57-59.

Öztürk, N., M.R. Ulusoy, L. Erkılıç \& S. Bayhan, 2004. Malatya ili kayısı bahçelerinde saptanan zararlılar ile avcı türler. Bitki Koruma Bülteni, 44 (1-49): 1-13.

Sönmezyıldız, H., 2006. Bartın Yöresinde Fidanlarda ve Süs Bitkilerinde Zarar Yapan Böcekler. Zonguldak Karaelmas Üniversitesi, Fen Bilimleri Enstitüsü, Yüksek Lisans Tezi, Zonguldak, 172 s.

Şahin, M., 2007. Kayseri Merkez Afit (Homoptera: Aphididae) Faunasının Belirlenmesi. Niğde Üniversitesi, Fen Bilimleri Enstitüsü, Yüksek Lisans Tezi, Niğde, 92 s.

Toper Kaygın, A., G. Görür \& F. Çota, 2008. Contribution to the Aphid (Homoptera : Aphididae) species damaging on woody plants in Bartın, Türkiye. International Journal of Engineering Science, 2 (1): 83-86.

Toper Kaygın, A., G. Görür \& F. Çota Sade, 2009. Aphid (Hemiptera: Aphididae) species determined on herbaceous and shrub plants in Bartin Province in Western Blacksea Region of Turkey. African Journal of Biotechnology, 8 (12): 2893-2897.

Toros, S., N. Uygun, R. Ulusoy, S. Satar \& I. Özdemir, 2002. Doğu Akdeniz Bölgesi Aphidoidea Türleri. Tarım ve Köyişleri Bakanlığı Tarımsal Araştırmalar Genel Müdürlüğü, Ankara. 108 s.

Toros, S., B. Yaşar, M. S. Özgökçe \& İ. Kasap, 1996. "Van ilinde Aphidoidea (Homoptera) üstfamilyasına bağlı türlerin saptanması üzerine çalışmalar, 549". Türkiye 3. Entomoloji Kongresi (24-28 Eylül, Ankara) Bildirileri, $716 \mathrm{~s}$.

Tuatay, N. \& G. Remaudière, 1964. Première contribution au catalogue des Aphididae (Hom.) de la Turquie. Revue de Pathologie Végétale et d' Entomologie Agricole de France, 43: 243-278.

Tuatay, N., 1988. Türkiye yaprakbitleri (Homoptera: Aphididae) I. Aphidinae Macropsophini (I. Kısım). Bitki Koruma Bülteni, 28 (1-2): 1-28.

Tuatay, N., 1990. Türkiye yaprakbitleri (Homoptera: Aphididae) II. Aphidinae Macropsophini (II. Kısım). Bitki Koruma Bülteni, 30 (1-4): 29-44.

Tuatay, N., 1991. Türkiye yaprakbitleri (Homoptera: Aphididae) III. Aphidinae: Macropsophini (III. Kısım). Bitki Koruma Bülteni, 31 (1-4): 3-18.

Tuatay, N., 1993. Türkiye yaprakbitleri (Homoptera: Aphididae) IV. Aphidinae: Aphidini (I. Kısım). Bitki Koruma Bülteni, 33 (3-4): 83-106.

Tuatay, N. 1999. Türkiye yaprakbitleri (Homoptera: Aphididae): V. Chaitophinae, Lachninae ve Thelaxinae. Bitki Koruma Bülteni, 39 (1-2): 1-21.

Tuatay, N., S. Gül, A. Demirtola, A. Kalkandelen \& N. Çağatay, 1967. Nebat Koruma Müzesi Kataloğu (1961-1966). T.C. Tarım Bakanlığı Zirai Mücadele Zirai Karantina Genel Müdürlüğü Yayınları, Mesleki Kitaplar Serisi. Ayyıldız Matbaası, Ankara, 66s.

Ural, İ., M. Işık \& A. Kurt, 1973. Doğu Karadeniz Bölgesi fındık bahçelerinde tesbit edilen böcekler üzerinde bazı incelemeler. Bitki Koruma Bülteni, 13 (2): 55-66. 
Uygun, N., S. Toros, M.R. Ulusoy, S. Satar \& I. Özdemir, 2001. Doğu Akdeniz Bölgesi Aphidoidea (Homoptera) Türleri ile Bunların Parazitoit ve Predatörlerinin Saptanması. Bilim ve Teknoloji Araştırma Kurumu Tarım ve Orman Araştırma Grubu, Proje No. TÜBITAK-TOGTAK 1720, Ankara, 214 s.

Ünal, S. \& E. Özcan, 2005. Kastamonu yöresi Aphididae (Homoptera) türleri. Süleyman Demirel Üniversitesi Orman Fakültesi Dergisi, A (1): 76-83.

Yıldırım, E. \& Z. Eroğlu, 2015. Atatürk Üniversitesi (Erzurum) yerleşkesinde odunsu bitkilerde bulunan zararlı böcek türleri. Atatürk Üniversitesi Ziraat Fakültesi Dergisi, 46 (1): 29-37.

Zeren, O., 1989. Çukurova Bölgesinde Sebzelerde Zararlı Olan Yaprakbitleri (Aphidoidea) Türleri, Konukçuları, Zararları ve Doğal Düşmanları Üzerinde Araştırmalar. Tarım Orman ve Köy işleri Bakanlığı Araştırma Yayınları Serisi Yayın No.59, 205 s. 